\title{
Wafer Surface Charge Reversal as a Method of Simplifying Nanosphere Lithography for Reactive Ion Etch Texturing of Solar Cells
}

\author{
Daniel Inns, Patrick Campbell, and Kylie Catchpole \\ Centre of Excellence for Advanced Silicon Photovoltaics and Photonics, University of New South Wales, \\ Sydney NSW 2052, Australia
}

Received 23 April 2007; Accepted 18 July 2007

Recommended by Armin G. Aberle

\begin{abstract}
A simplified nanosphere lithography process has been developed which allows fast and low-waste maskings of Si surfaces for subsequent reactive ion etching (RIE) texturing. Initially, a positive surface charge is applied to a wafer surface by dipping in a solution of aluminum nitrate. Dipping the positive-coated wafer into a solution of negatively charged silica beads (nanospheres) results in the spheres becoming electrostatically attracted to the wafer surface. These nanospheres form an etch mask for RIE. After RIE texturing, the reflection of the surface is reduced as effectively as any other nanosphere lithography method, while this batch process used for masking is much faster, making it more industrially relevant.
\end{abstract}

Copyright (C) 2007 Daniel Inns et al. This is an open access article distributed under the Creative Commons Attribution License, which permits unrestricted use, distribution, and reproduction in any medium, provided the original work is properly cited.

\section{INTRODUCTION}

Multicrystalline Si wafers make up the majority of the photovoltaic market. Due to the variety of grain orientations, they cannot be textured cheaply by the anisotropic wet chemical etches used for monocrystalline silicon. One possibility for texturing is reactive ion etching (RIE). RIE can randomly texture a Si surface, with the minimum reflectance of below $5 \%$ achieved vcommentWe changed "masking" to "maskings" for the sake of clarity. Please check. $[1,2]$. This maskless RIE requires the etching of several microns of $\mathrm{Si}$ and has the resulting long process times. The use of a mask during RIE allows greater control over the surface topography than maskless RIE and results in shallower textures with reduced etch times. The best reflection achieved to date using an RIE mask applied a photolithography process to form pyramids [3], with minimum reflectances around 5\% achieved.

As an alternate to a photolithography mask, RIE masks can be formed by nanospheres. Nanospheres are highly uniform spheres (typically of silica or polystyrene) and are commercially available and very cheap. In previous work with nanosphere masks, the mask has been formed by spincoating, resulting in a uniform monolayer of spheres across the surface. These particles are then the etch mask for subsequent RIE [4]. Without an antireflection coating, the mini- mum reflectance is reduced around 13\% by etching less than $500 \mathrm{~nm}$ of $\mathrm{Si}-$ making the process fast and high-throughput compared to other RIE textures, with or without masks.

Spinning-on of the nanosphere mask requires very careful control of the nanoparticle solution used, with many critical factors including spin speed, substrate surface treatments, and particulate concentration [5]. The typically rough wafer surface complicates spinning as some large regions are left unmasked [4]. Additionally, the throughput would be low and the masking solution wastes high for a factory application. A solution with a high nanosphere solids content is required for this spinning process and accentuates the waste problem.

Dipping a wafer into a masking solution would be attractive in a manufacturing environment, as the batch process could have very high throughput. Dipping could also coat large area wafers, $1 \mathrm{~m}^{2}$ glass sheets, or other objects which are not suitable for spinning due to size, asymmetry, or weight. For some applications, it would also be an advantage to mask both sides of a wafer at once, to achieve a double-sided texture. Silicon dioxide, which comprises the surface of the standard wafer and the silica beads commonly used for the masking, has a negative surface charge when immersed in a solution with a $\mathrm{pH}$ above its isoelectric point of $\sim 2.5$ [6]. To electrostatically attract the negative spheres to the negative wafer 


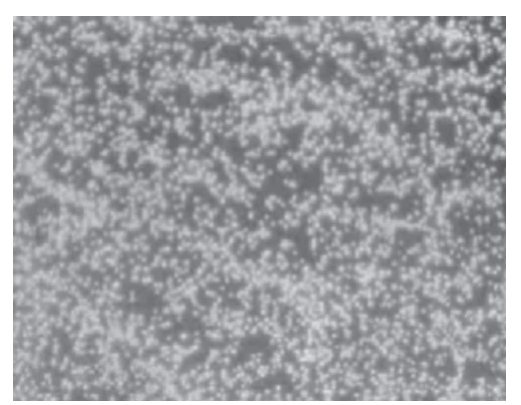

Figure 1: Dark field optical microscope image of the nanosphere mask applied to a wafer by reversing its surface charge. The image is $180 \times 160 \mu \mathrm{m}$.

surface, the surface charge of the wafer must be reversed by the application of a positive coating.

This work shows that a simple dipping process can reverse the surface charge of a wafer, and another dip can mask the surface. Masking based on this idea can lead to high-nanosphere surface coverage, and etching through the nanosphere masks is well controlled by RIE. A variety of textures can be formed and very good reflectance reduction achieved. The geometry of the texture features can be altered by changing RIE processing parameters (primarily pressure and gas mixtures), which also controls the etch rate of the nanospheres that act as the mask.

\section{EXPERIMENTS AND DISCUSSION}

Below is outlined the process used to optimize the two solutions used for dip coating wafers, first for wafer surface charge reversal, followed by electrostatic attraction of a nanosphere lithography mask. Afterwards, some RIE textured surfaces are examined by SEM and the reflectance of the best texture assessed.

\subsection{Reversing the surface charge of silicon wafers}

Layer-by-layer coating can be used to build up a variety of types of films, by alternate deposition of positive followed by negative ions, molecules, or colloids [7]. The use of colloidal particles such as nanospheres in the layer-by-layer method was pioneered by Iler [8]. In this work, Iler's technique was used to deposit a single bilayer of hydrolyzed $\mathrm{Al}^{+}$ions followed by negatively charged $\mathrm{SiO}_{2}$ nanospheres. The composition of the $\mathrm{Al}^{+}$solution and the $\mathrm{pH}$ of the nanosphere solution were adjusted to optimize the mask coatings.

Before any surface treatments, a polished and oxide-free $\mathrm{Cz}$ Si wafer surface was oxidized by a 5 -minute piranha clean $\left(\mathrm{H}_{2} \mathrm{SO}_{4}: \mathrm{H}_{2} \mathrm{O}_{2}\right.$ solution, $2: 1$ mixture) and then rinsed for 10 minutes. A polished surface was chosen as its predictably high reflection is best for quantifying the benefits of the resulting texture. In order to attach a positive $\mathrm{Al}$ coating to the wafer, a set of $\mathrm{AlNO}_{3}$ solutions (concentration varying from $400-800 \mu \mathrm{M}$ ) was prepared, and small amounts of $50 \mathrm{mM}$ $\mathrm{NaOH}$ were added to adjust the $\mathrm{pH}$ and concentration of the solutions. The cleaned wafer was dipped into the solution

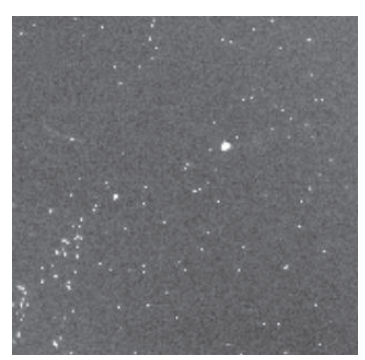

(a)

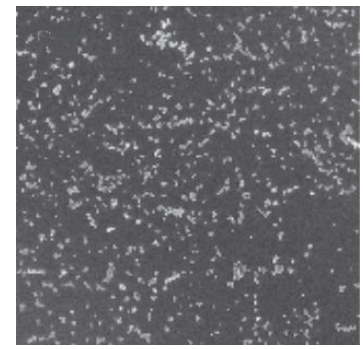

(b)

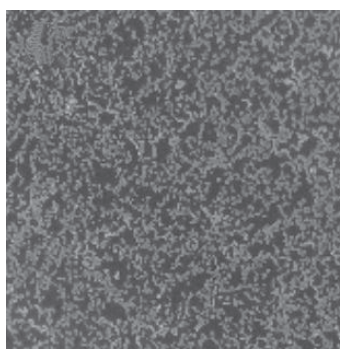

(c)

FIGURE 2: SEM images of the nanosphere masks attached to a silicon wafer surface using 3 different $\mathrm{pH}$ masking solutions: (a) $\mathrm{pH}=4$, (b) $\mathrm{pH}=3.5$, (c) $\mathrm{pH}=3.2$. The images are $80 \mu \mathrm{m} \times 80 \mu \mathrm{m}$.

for 30 seconds to allow adsorption of a positive layer. Immediately afterwards, a 10 seconds dip-in-deionized water was followed by a nitrogen gas blow dry to remove excess liquid.

To test the effectiveness of the positive coating solutions, the positive coated wafers were dipped in a masking solution of negative nanospheres. The nanospheres used in this study are $400 \mathrm{~nm}$ diameter silica beads from Bangs Labs, product code SS02N, which comes in a colloid solution with $10 \%$ by weight nanospheres. A $3.5 \mathrm{pH}$ solution of acetic acid was used, containing $2 \%$ by weight nanospheres. A $3.5 \mathrm{pH}$ was chosen as it is greater than the isoelectric point of $\mathrm{SiO}_{2}$ and much less than the isoelectric point of $\mathrm{Al}$ and $\mathrm{Al}_{2} \mathrm{O}_{3}$ (the composition of the positive coating layer), giving opposite charged surfaces.

Optical microscope imaging in dark field mode revealed that a combination of $650 \mu \mathrm{M} \mathrm{AlNO}$ with $125 \mu \mathrm{M} \mathrm{NaOH}$ resulted in the most dense coating of nanospheres onto the wafer. This mixture of chemicals gives the mask solution a $\mathrm{pH}$ of 4.1. A picture of the surface is shown in Figure 1. It should be reiterated at this stage that an oxide was formed on the wafer by a piranha clean. Vastly, different nanosphere masks result when using a wafer which has a different surface, 


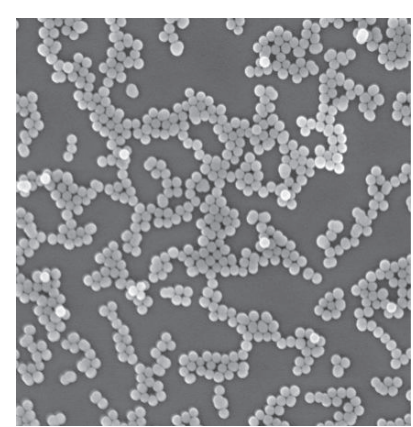

(a)

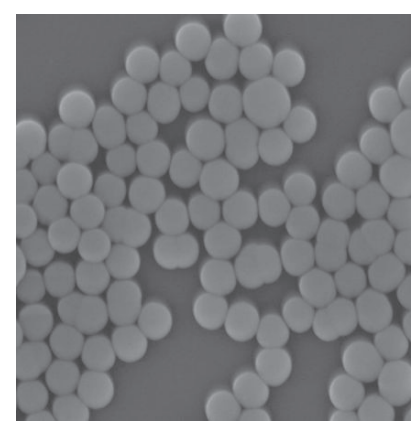

(b)

FIGURE 3: SEM images of the nanosphere coatings from the $3.2 \mathrm{pH}$ masking solution. Scale can be taken from the $400 \mathrm{~nm}$ diameter sphere diameters.

such as a thick thermal oxide, native oxide, or no oxide at all. In addition, masking of multicrystalline wafers may require a slightly different composition due to their surface roughness. The solutions used here must be optimized for each type of surface, and each application.

\subsection{Electrostatically attaching nanosphere masks}

Varying the $\mathrm{pH}$ of the masking solution strongly alters the surface charge of the solids in solution and hence influences the electrostatic attraction between the positive coated wafer and the negative nanospheres. Mixtures of $50 \mathrm{mM}$ acetic acid and $50 \mathrm{mM} \mathrm{NaOH}$ were used to tailor the $\mathrm{pH}$ of the nanosphere solutions. After $\mathrm{pH}$ was determined, a small volume of nanospheres was added to make the solution $2 \%$ by weight nanospheres. As expected for such a buffer solution, this amount of dilution with nanosphere solution was found to have negligible effect on the $\mathrm{pH}$. The solution $\mathrm{pH}$ was varied upwards of 3.2 by this process, and the nanosphere masks examined by SEM. Figure 2 shows the nanosphere masks achieved.

The lowest $\mathrm{pH}$ gives a denser mask, covering more of the surface. SEM images of 2 different areas of the best mask can be seen in Figure 3. Around $60 \%$ of the surface is masked with large $2 \mathrm{D}$ nanosphere arrays, with unmasked regions usually closer than 3 sphere diameters from a nanosphere. Stronger concentrations of acetic acid were used to achieve a lower $\mathrm{pH}$, but the masks were very sparse and gave poorer surface coverage than that shown in Figure 3. This may have

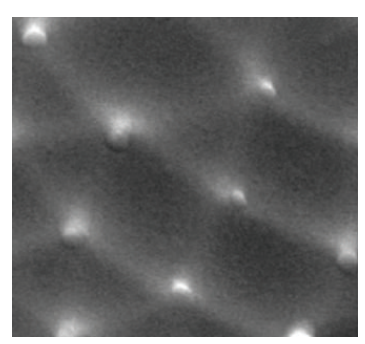

(a)

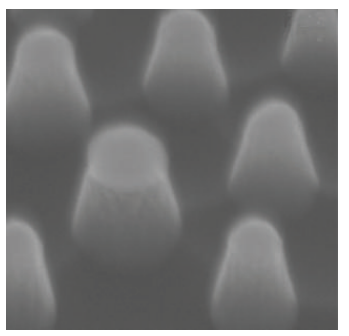

(b)

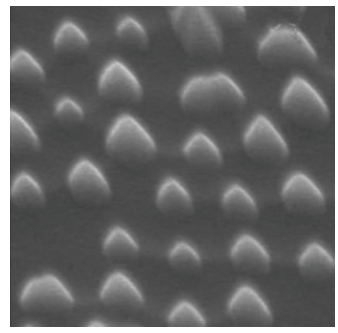

(c)

FIGURE 4: SEM images (taken on a $45^{\circ}$ tilt) of different RIE textures made using $\mathrm{SF}_{6}$ plasmas during RIE. (a) Shallow honeycomb feature. (b) Flat-topped cones, with best antireflection properties. (c) Small pointed structures.

been because the nanosphere surface charge/wafer surface charge was not well balanced, or the positive coating partially dissolved in the stronger acid. Other acids are likely to give different results, as they will have a different $\mathrm{pH}$ at this $50 \mathrm{mM}$ concentration, and they will interact differently with the positive wafer coating. A denser or more homogeneous coating than that shown in Figure 3 may be possible using the surface charge reversal masking process. All previous work on nanosphere lithography has assumed a requirement for a continuous monolayer mask, although the merits of this have not been demonstrated. Using these simple-to-apply lithography masks and then forming an RIE texture, the reflection is shown to be as good as anything achieved using a spun on, more homogeneous monolayer mask.

\subsection{RIE texturing results}

RIE is commonly used in the microelectronic industries and the equipment appears in many labs and photovoltaics manufacturing plants where it is used for edge isolation. A handful of different textures were created by RIE using $\mathrm{SF}_{6}$ gas and the best mask as shown in Figure 3. Figure 4 shows some different shaped features that were created. The surfaces were 


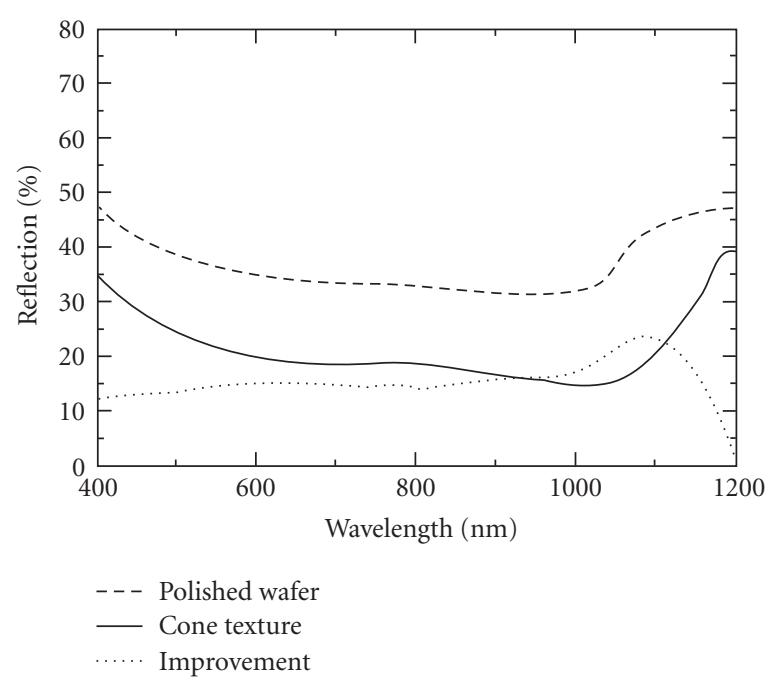

FIgURE 5: Reflectance of a polished silicon wafer, and an RIE textured wafer using the simplified nanosphere lithography mask. The difference between the two is also shown.

etched with different $\mathrm{SF}_{6}$ pressures and flow rates. The shallow honeycomb features in Figure 4(a) were made with a process pressure of $6.9 \mathrm{~Pa}$ in 10 minutes. The flat-topped cone-shaped texture from Figure 4(b) has the best antireflection effect, and was made using the highest process pressure of $7.5 \mathrm{~Pa}$ in 4 minutes. The small pointed structures in Figure 4 (c) are made with $3.5 \mathrm{~Pa}$ and took $8 \mathrm{~min}$. On the regions where there were no nanospheres, the surface is gently curved and almost flat.

After texturing, the wafers were dipped into a solution of $5 \% \mathrm{HF}$ which removed the remains of the nanospheres and removed the oxide from the silicon surface. The hemispherical reflectance of the silicon surfaces were then measured using an integrating sphere accessory with a Varian CARY 500 spectrophotometer. As the small RIE system used in this study only etches uniformly across a $3 \mathrm{~cm}$ diameter area, only the central region of the wafer was measured. Figure 5 shows that the cone texture performs well across the full wavelength range, with a minimum reflectance of $14.5 \%$.

\section{CONCLUSION}

Nanospheres are a suitable etch mask for RIE texturing. The masks are usually spun on, but this is a slow serial process and wastes a great deal of nanosphere solution. By reversing the surface charge of a polished silicon wafer, the mask has been applied by simply dipping into a dilute acidic solution of nanospheres. This process is suitable for a manufacturing environment, as the masking and drying process takes only 2 minutes, and the RIE texturing (which uses standard equipment) takes only 4 minutes.

The nanosphere masks used do not fully coat the surface in a homogeneous monolayer as seen in spun-on masks. The mask is more like a random arrangement of tendrils, stretching over the wafer surface in $2 \mathrm{D}$ arrays. This has proven to be no disadvantage, as the minimum front sur- face reflectance produced with this process is as low as has been achieved by any other nanosphere lithography masking process [4]. The added advantage of the masking process developed here is the increased processing speed that should translate to factory throughput and cost savings. The application for these colloidal masks stretches beyond polished silicon wafers, as the simple dipping process could also create nanosphere coatings onto very large multicrystalline wafers or to heavy glass sheets, which would not be suitable for spinning.

\section{ACKNOWLEDGMENTS}

Parts of this work were supported by the Australian Research Council (ARC) via its Centres of Excellence scheme. The first author acknowledges a faculty of engineering Ph.D. scholarship from UNSW. The third author has an Australian Research Council Postdoctoral Fellowship.

\section{REFERENCES}

[1] Y. Inomata, K. Fukui, and K. Shirasawa, "Surface texturing of large area multicrystalline silicon solar cells using reactive ion etching method," Solar Energy Materials and Solar Cells, vol. 48, no. 1-4, pp. 237-242, 1997.

[2] D. S. Ruby, S. H. Zaidi, S. Narayanan, B. M. Damiani, and A. Rohatgi, "Rie-texturing of multicrystalline silicon solar cells," Solar Energy Materials and Solar Cells, vol. 74, no. 1-4, pp. 133137, 2002.

[3] S. Winderbaum, O. Reinhold, and F. Yun, "Reactive ion etching (RIE) as a method for texturing polycrystalline silicon solar cells," Solar Energy Materials and Solar Cells, vol. 46, no. 3, pp. 239-248, 1997.

[4] W. A. Nositschka, C. Beneking, O. Voigt, and H. Kurz, "Texturisation of multicrystalline silicon wafers for solar cells by reactive ion etching through colloidal masks," Solar Energy Materials and Solar Cells, vol. 76, no. 2, pp. 155-166, 2003.

[5] H. W. Deckman and J. H. Dunsmuir, "Natural lithography," Applied Physics Letters, vol. 41, no. 4, pp. 377-379, 1982.

[6] R. Hunter, Zeta Potential in Colloid Science: Principles and Applications, Academic Press, London, UK, 1981.

[7] P. T. Hammond, "Form and function in multilayer assembly: new applications at the nanoscale," Advanced Materials, vol. 16, no. 15, pp. 1271-1293, 2004.

[8] R. K. Iler, "Multilayers of collodal particles," Journal of Colloid Interface Science, vol. 21, no. 6, pp. 569-594, 1966. 

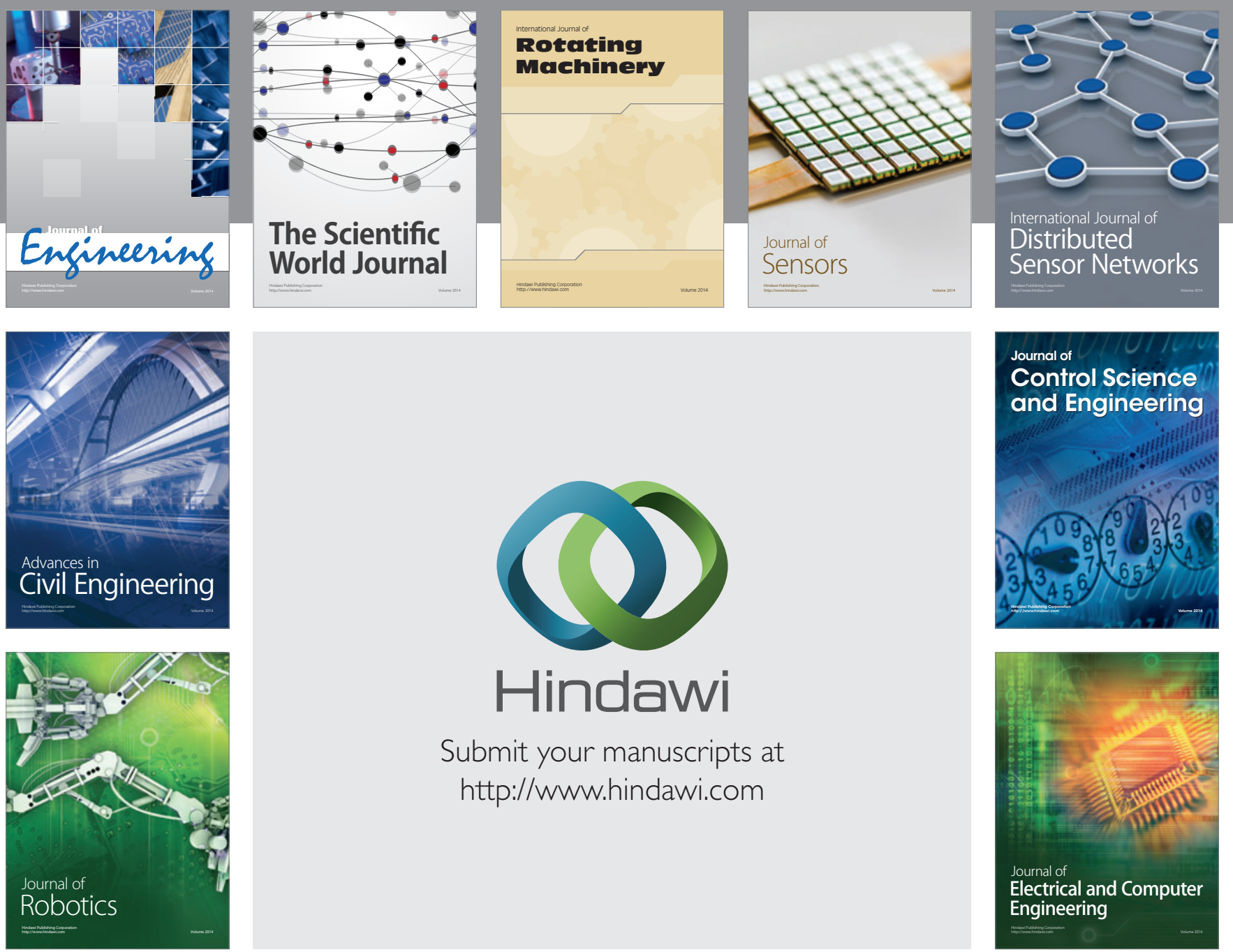

Submit your manuscripts at

http://www.hindawi.com
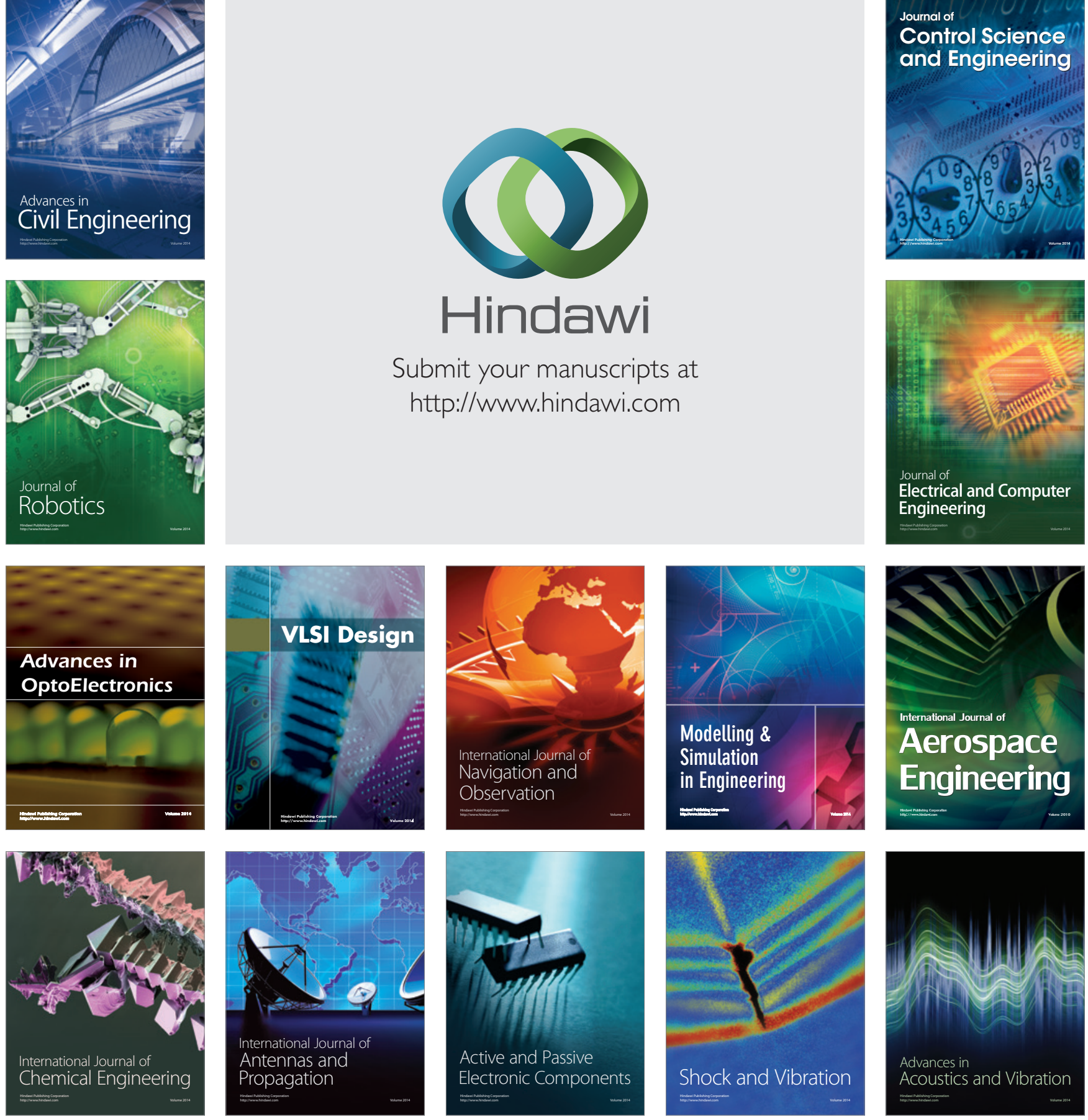